\title{
ASO Author Reflections: Seizing the Momentum to Diversify Cancer Clinical Trial Enrollment
}

\author{
Mariam F. Eskander, MD, MPH ${ }^{1,2}$, Samilia Obeng-Gyasi, MD, MPH ${ }^{1}$, and Allan Tsung, MD ${ }^{1}$ \\ ${ }^{1}$ The Arthur G. James Cancer Hospital and Solove Research Institute at the Ohio State University Wexner Medical Center, \\ Columbus, OH; ${ }^{2}$ Rutgers Cancer Institute of New Jersey, New Brunswick, NJ
}

\section{PAST}

It is easy to forget clinical trial enrollment when considering the impact of social determinants of health. Clinical trials offer patients other, sometimes superior, options for cancer treatment. They drive progress and lead to breakthroughs for deadly diseases. However, access to clinical trials is starkly divided along geographic, racial and economic lines. ${ }^{1}$

At present, patients enrolled in clinical trials do not represent the racial, ethnic, or socioeconomic diversity of the U.S. population. Although people of color and those of low socioeconomic status bear a disproportionate burden of cancer incidence and mortality in this country, they make up a small minority of trial participants. ${ }^{2}$ Lack of representation limits generalizability and impedes the discovery of therapeutic targets in different populations, further exacerbating inequities. Understanding barriers to enrollment is critically important for the development of both policy changes and on-the-ground interventions.

\section{PRESENT}

Using a national database, Eskander et al. found pronounced socioeconomic disparities in clinical trial enrollment for pancreatic cancer and minimal progress in increasing the representation of low-income and black patients over the study period of $2004-2016 .^{3}$ Of all

(C) Society of Surgical Oncology 2021

First Received: 27 September 2021

Accepted: 28 September 2021;

Published Online: 16 October 2021

A. Tsung, MD

e-mail: allan.tsung@osumc.edu patients enrolled in clinical trials, only $4-6 \%$ were black and $3-5 \%$ were Medicaid-insured. This rate did not change significantly over time.

After adjusting for clinical and tumor characteristics, race, insurance status, neighborhood education level, and geography were predictors of clinical trial enrollment. Nonwhite patients had half the odds of enrolling as white patients. Compared with having private insurance, underinsured patients had approximately $60 \%$ lower odds, as were patients living in the South as opposed to the Northeast. The highest quartile of neighborhood education was associated with twice the odds of enrollment as the lowest. In contrast, patients treated at academic centers had six times the odds of enrolling as those treated at nonacademic centers.

\section{FUTURE}

Change is possible but requires planning and effort, as seen in the recent campaign to increase diversity in COVID-19 vaccine trials. ${ }^{4}$ Transparency regarding the race/ethnicity and socioeconomic status of clinical trial participants is only the first step. ${ }^{5}$ At a national level, insurance barriers are slowly being chipped away. The Clinical Treatment Act, passed in 2020 and effective in 2022, holds promise for increasing access for Medicaid beneficiaries.

A myriad of structural factors remain, hindering the path to equity. Efforts to increase enrollment should address:

- Language barriers

- Health literacy issues

- The financial burden of travel

- Lack of trial opportunities at community and safety-net hospitals

- Mistrust of the healthcare system due to institutional and interpersonal racism and classism 
- Lack of racial and ethnic diversity in the oncology workforce

Education is imperative, both for providers and patients. In addition to increasing diversity on our oncology teams, we must recognize the role of implicit bias in the recruitment of patients from historically marginalized and minoritized populations. Furthermore, education efforts regarding the benefits of and availability of clinical trials should target historically disenfranchised populations and involve community members. Hospitals should leverage social workers, case managers, and patient navigators to address barriers rooted in health-related social needs to facilitate the enrollment of racially and economically diverse populations.

The problem is thorny and complex, but we must not squander existing momentum around health equity. Each of us holds a responsibility to our patients to ensure equal access to clinical trials.

DISCLOSURES None.

\section{REFERENCES}

1. Murthy VH, Krumholz HM, Gross CP. Participation in cancer clinical trials: race-, sex-, and age-based disparities. JAMA. 2004;291(22):2720-6. https://doi.org/10.1001/jama.291.22.2720.

2. Vose J. Minority enrollment to clinical trials: road to increased access. Oncology (Williston Park). 2021;35(3):107. https://doi.org/ 10.46883/onc.2021.3503.0107.

3. Eskander M, Gil L, Beal E, et al. Access denied: inequities in clinical trial enrollment for pancreatic cancer. Ann Surg Oncol. 2021. https://doi.org/10.1245/s10434-021-10868-4.

4. Jaklevic MC. Researchers strive to recruit hard-hit minorities into COVID-19 vaccine trials. JAMA. 2020;324(9):826-8. https://doi. org/10.1001/jama.2020.11244.

5. Herremans KM, Riner AN, Winn RA, Trevino JG. Diversity and inclusion in pancreatic cancer clinical trials. Gastroenterology. 2021. https://doi.org/10.1053/j.gastro.2021.06.079.

Publisher's Note Springer Nature remains neutral with regard to jurisdictional claims in published maps and institutional affiliations. 